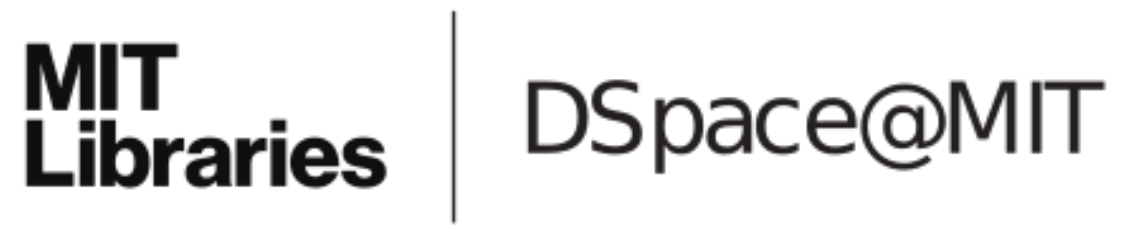

MIT Open Access Articles

Vortex-induced vibration of a linearly sprung cylinder with an internal rotational nonlinear energy sink in turbulent flow

The MIT Faculty has made this article openly available. Please share how this access benefits you. Your story matters.

As Published: https://doi.org/10.1007/s11071-019-04775-3

Publisher: Springer Netherlands

Persistent URL: https://hdl.handle.net/1721.1/131785

Version: Author's final manuscript: final author's manuscript post peer review, without publisher's formatting or copy editing

Terms of Use: Article is made available in accordance with the publisher's policy and may be subject to US copyright law. Please refer to the publisher's site for terms of use. 


\section{Vortex-induced vibration of a linearly sprung cylinder with an internal rotational nonlinear energy sink in turbulent flow}

Cite this article as: Antoine Blanchard, Lawrence A. Bergman and Alexander F. Vakakis, Vortex-induced vibration of a linearly sprung cylinder with an internal rotational nonlinear energy sink in turbulent flow, Nonlinear Dynamics https://doi.org/10.1007/s11071-01904775-3

This Author Accepted Manuscript is a PDF file of an unedited peer-reviewed manuscript that has been accepted for publication but has not been copyedited or corrected. The official version of record that is published in the journal is kept up to date and so may therefore differ from this version.

Terms of use and reuse: academic research for non-commercial purposes, see here for full terms. https://www.springer.com/aam-terms-v1 


\title{
Vortex-Induced Vibration of a Linearly-Sprung Cylinder with an Internal Rotational Nonlinear Energy Sink in Turbulent Flow
}

\author{
Antoine Blanchard · Lawrence A. Bergman · Alexander F. Vakakis \\ This work is dedicated to the memory of Prof. Ali H. Nayfeh, outstanding educator, respected teacher, admired scholar.
}

Received: date / Accepted: date

\begin{abstract}
We computationally investigate three-dimensional flow past a linearly-sprung cylinder undergoing vortex-induced vibration (VIV) transverse to the free stream and equipped with an internal dissipative rotational nonlinear energy sink (NES). The rotational NES consists of a line mass allowed to rotate at constant radius about the cylinder axis, with linearly damped rotational motion. We consider a value of the Reynolds number $(R e=10000$, based on the cylinder diameter and free-stream velocity) at which flow past a linearlysprung cylinder with no NES is three-dimensional and fully turbulent. For this $R e$ value, we show that the rotational NES is capable of passively harnessing a substantial amount of kinetic energy from the rectilinear motion of the cylinder, leading to significant suppression of cylinder oscillation and a nearly two-fold reduction in drag. The results presented herein are of potentially practical significance since they demo strate a novel passive mechanism for VIV suppression and drag reduction in a high- $R e$ bluff-body flow, and lay down the groundwork for designing nonlinear energy sinks with a view to enhancing the performance of VIV-induced power generation in marine currents.
\end{abstract}

Keywords Vortex-induced vibration - Nonlinear energy sink $\cdot$ Energy harvesting $\cdot$ Turbulent vortex shedding

\footnotetext{
A. Blanchard $(\bowtie)$

Department of Aerospace Engineering, University of Illinois at Urbana-Champaign, Urbana, IL 61801

E-mail: ablancha@mit.edu

Present address: Massachusetts Institute of Technology

\section{A. Bergman}

Department of Aerospace Engineering, University of Illinois at Urbana-Champaign, Urbana, IL 61801

A. F. Vakakis

Department of Mechanical Science and Engineering, University of Illinois at Urbana-Champaign, Urbana, IL 61801
}

\section{Introduction}

Vortex-induced vibration (VIV) of a rigid circular cylinder is of practical importance in a range of applications involving flow past bluff bodies. Examples include tall buildings and chimneys, heat-exchanger tubes in nuclear power plants, power transmission lines, and off-shore structures. In these cases, and in the vast majority of engineering applications, large-amplitude VIV can cause severe damage to, and even failure of, the structure. This has led to a large number of fundamental studies devoted to understanding how the motion of a linearly-sprung cylinder is coupled to the dynamics of the wake, including the alternate shedding of vortices that gives rise to, and interacts with, the VIV of the cylinder. For recent reviews on the subject, we refer the reader to - Williamson \& Govardhan [1] and Païdoussis et al. [2].

In addition to active and passive VIV suppression, which has been a subject of active research in the last few decades [3-5], there is also growing interest in harvesting energy from VIV in submarine flows to generate electrical power. From that perspective, the effects of VIV can be beneficial, since they can be incorporated into designs to achieve efficient harvesting of kinetic energy from the fluid motion. This is why focus has also been directed toward enhancing the flow-induced vibrations to realize large-amplitude motion of the structure that, in turn, can lead to efficient energy extraction [6-9]. A key advantage of the vibrating-cylinder approach is that there are no sharp edges or rotating blades, as opposed to axial-flow horizontal-axis turbines [10]. This approach thus poses much less threat to marine life, and has no high-aspect-ratio rotating elements that can be damaged by impact, cavitation, or fatigue.

One of the premier experimental investigations on this subject was performed by Bernitsas and colleagues [6,7], who used the rectilinear motion of the cylinder to move a magnet attached to the cylinder past a conducting coil to 
generate an electrical current. Despite being relatively successful, this approach, dubbed "Vortex Induced Vibration Aquatic Clean Energy" (VIVACE), suffers from at least two shortcomings. First, at each end of its travel, the velocity of the magnet relative to the fixed coil is zero, resulting in significant intermittency in the electrical current produced. Second, for the cylinder-to-fluid density ratios so far investigated, the VIVACE approach works well in a relatively narrow range of Reynolds numbers ( $R e$ between 20000 and 30000 , based on the cylinder diameter and free-stream velocity) where the VIV amplitude is significantly higher compared to other ranges of $R e$. This leads to significantly reduced efficiency during periods when the Reynolds number is not in that range.

An alternative option explored recently is to consider use of a fully passive, internal, nonlinear energy sink (NES) to harvest energy from the motion of the oscillating cylinder and, indirectly, the surrounding fluid. An NES consists of a mass whose motion is coupled to that of the primary structure in an essentially nonlinear fashion (meaning, a stiffness connection which has a purely nonlinear characteristic and cannot be linearized), and retarded by a linear viscous damper [11]. Essentially nonlinear coupling between the structure and the NES, in conjunction with dissipation, allows for passive, nearly unidirectional "targeted energy transfer" (TET) from the structure to the NES, where it is trapped and dissipated [11]. Use of an NES for passive mitigation of large-amplitude structural vibrations has been successful in a variety of applications, including suppression of aeroelastic instabilities in an aircraft wing [12], blast and seismic mitigation [13,14], and acoustic isolation [15]. The NES can also be combined with a piezoelectric [16-18], magnetostrictive [19], or electromagnetic [20-22] energyharvesting element to collect vibration energy from the structure through the TET mechanism. In contrast to the classical linear vibration absorber (LVA) [23], which is tuned to a single excitation frequency, the NES, because of its essentially nonlinear nature, is much more robust to large variations in the frequency of the excitation, and detuning of the natural frequencies of the structure. This means that the NES is generally the broadband device of choice to harvest energy from impulse or random excitations, as it achieves much better efficiency and robustness in performance compared to the classical LVA [23].

In the context of VIV, it is only recently that the concept of NES began to gain traction, with the work of Tumkur and colleagues [24-26]. They were the first to show that a translational NES (consisting of a mass allowed to translate along the axis of travel of the cylinder and whose rectilinear motion is retarded by an essentially cubic spring and a linear viscous damper) can be used to passively mitigate VIV of a linearly-sprung cylinder at low Reynolds numbers (between 20 and 120, in which range the flow is expected to be two- dimensional and laminar), vastly outperforming the linear vibration absorber [24,25]. In the same $R e$ range, Tumkur et al. [26] and Blanchard et al. [27,28] showed that coupling a rotational NES (consisting of a mass allowed to rotate about the cylinder axis, and a damper that linearly retards the rotational motion of that mass) to the oscillating cylinder gives rise to a variety of remarkable phenomena not seen in NESless VIV. They found situations in which the NES leads to significant passive VIV suppression, partial stabilization of the wake, considerable drag reduction, coexistence of multiple long-time solutions, and resonance captures on slowinvariant manifolds. Others have since considered a variety of NES-equipped VIV configurations [29-31], and found further evidence that an NES can significantly alter the VIV and the wake.

The results of Blanchard et al. [27] demonstrated the existence of a novel mechanism for energy transfer-besides TET - when the NES interacts with an infinite-dimensional system (the fluid flow in [27]). The authors showed that the indirect interaction of the rotational NES with the flow (mediated by the cylinder motion) can give rise to significant energy redistribution among fluid modes, which in turn leads to considerable elongation and symmetrization of the wake. The mechanism was shown to be gradual NES-induced redistribution of energy from high- to low-energy fluid modes, leaving only the mean (symmetric) mode as the dominant one. So in addition to TET and energy dissipation, the NES led to substantial energy redistribution among the coherent structures of the surrounding fluid. This finding is significant because it suggests the existence of potentially unexplored nonlinear energy transfer mechanisms in situations where a strongly nonlinear low-dimensional oscillator interacts with an infinite-dimensional system.

The present work is the first of its kind to establish the potential of a rotational NES for use as an energy harvesting and vibration suppression device at values of the Reynolds number (on the order of 10000) for which flow past an NESless linearly-sprung cylinder is three-dimensional and fully turbulent. In addition to appealing harvesting and suppression properties, we find that the NES leads to substantial passive drag reduction, which is critical in a large number of bluff-body applications. The results pertain to a density ratio of ten, thus facilitating experiments in water and other liquids. The remainder of the paper is structured as follows. In $\S 2$, we formulate the fluid-structure interaction problem, and describe our computational approach. In $\S 3$, we present evidence that a rotational NES can serve as an efficient hydrokinetic energy harvesting and VIV suppression device in turbulent flow. In $\S 4$, we discuss connections between the solutions found in $\S 3$ and those reported previously by Blanchard et al. [27] at lower Re. We offer some conclusions in $\S 5$. 


\section{Formulation of the problem}

\subsection{Physical model}

We consider an incompressible fluid of constant density $\rho_{\mathrm{f}}$ and kinematic viscosity $v$ flowing with uniform velocity $U \mathbf{e}_{x}$ past an infinitely long, three-dimensional, rigid, circular cylinder of mass $M_{\text {cyl }}$ per unit length, and diameter $D$. The cylinder is allowed to translate transversely to the free stream, and its rectilinear motion is restrained by a spring of constant stiffness $K_{\text {cyl }}$ per unit length. To the cylinder is attached a rotational NES consisting of a line mass $M_{\mathrm{NES}}$ per unit length allowed to rotate at constant radius about the cylinder axis. The rotational NES lies a constant distance $r_{0}$ from the cylinder axis, and its rotational motion is linearly retarded by a rotational viscous damper with dissipative constant $C_{\mathrm{NES}}$ per unit length. Inertial coupling between the rectilinear motion of the cylinder and the rotational motion of the NES allows energy to be transferred from the former to the latter, part of which is dissipated by the linear rotational viscous damper. There is no direct coupling between the NES and the flow, so interaction of the former with the latter is necessarily mediated by the cylinder motion. The absence of a gravitational force acting on the cylinder and the NES mass is tantamount to the cylinder axis being mounted vertically. A schematic of the system is shown in Fig. 1.

The flow is governed by the incompressible Navier-Stoke equations,

$$
\begin{aligned}
& \frac{\partial \mathbf{v}}{\partial \tau}+\mathbf{v} \cdot \nabla \mathbf{v}=-\nabla p+\frac{1}{R e} \nabla^{2} \mathbf{v} \\
& \nabla \cdot \mathbf{v}=0
\end{aligned}
$$

where we have defined a dimensionless time $\tau=t U / D$, and scaled the length, velocity $\mathbf{v}$ and pressure $p$ by $D, U$ and $\rho_{\mathrm{f}} U^{2}$, respectively. The Reynolds number $R e=U D / v$ is based on the diameter of the cylinder and the free-stream velocity. The equations governing the rectilinear motion of the cylinder and the rotational motion of the NES mass are given by

$$
\begin{aligned}
& \frac{\mathrm{d}^{2} y_{1}}{\mathrm{~d} \tau^{2}}+\left(2 \pi f_{\mathrm{n}}^{*}\right)^{2} y_{1}=\varepsilon \bar{r}_{0} \frac{\mathrm{d}}{\mathrm{d} \tau}\left[\frac{\mathrm{d} \theta}{\mathrm{d} \tau} \sin \theta\right]+\frac{2 C_{\mathrm{L}}}{\pi m^{*}}, \\
& \frac{\mathrm{d}^{2} \theta}{\mathrm{d} \tau^{2}}+\lambda \frac{\mathrm{d} \theta}{\mathrm{d} \tau}=\frac{\sin \theta}{\bar{r}_{0}} \frac{\mathrm{d}^{2} y_{1}}{\mathrm{~d} \tau^{2}},
\end{aligned}
$$

where $y_{1}=Y / D$ is a dimensionless cylinder displacement, and $\theta$ is the angular position of the NES mass. We take $\theta=0$ to coincide with the positive $y$-axis. The other dimensionless parameters are the ratio $m^{*}=\rho_{\mathrm{b}} / \rho_{\mathrm{f}}$ of the density of the NES-equipped cylinder to that of the fluid, a dimensionless spring constant $f_{\mathrm{n}}^{*}=D \sqrt{K_{\mathrm{cyl}} /\left(M_{\mathrm{cyl}}+M_{\mathrm{NES}}\right)} /(2 \pi U)$, the ratio $\varepsilon=M_{\mathrm{NES}} /\left(M_{\mathrm{cyl}}+M_{\mathrm{NES}}\right)$ of the NES mass to the total mass of the cylinder (the nonrotating part and the attached rotating mass), a dimensionless radius $\bar{r}_{0}=r_{0} / D$, and a dimensionless damping coefficient $\lambda=\left(D C_{\mathrm{NES}}\right) /\left(U r_{0}^{2} M_{\mathrm{NES}}\right)$. In (2a), the lift force $F_{\mathrm{L}}$, which couples the rigid-body motion to the flow, has been scaled in favor of the lift coefficient $C_{\mathrm{L}}=2 F_{\mathrm{L}} /\left(\rho_{\mathrm{f}} U^{2} D\right)$. We note that, in terms of dimensionless flow quantities, the lift and drag coefficients can be expressed as

$C_{\mathrm{L}}=\int_{\Gamma_{\mathrm{cyl}}}\left(\boldsymbol{\sigma} \cdot \mathbf{n}_{\mathrm{cyl}}\right) \cdot \mathbf{e}_{y} \mathrm{~d} \Gamma$

and

$C_{\mathrm{D}}=\int_{\Gamma_{\mathrm{cyl}}}\left(\boldsymbol{\sigma} \cdot \mathbf{n}_{\mathrm{cyl}}\right) \cdot \mathbf{e}_{x} \mathrm{~d} \Gamma$,

respectively, where $\boldsymbol{\sigma}$ is the dimensionless stress tensor, and $\mathbf{n}_{\text {cyl }}$ is the outward unit normal to the cylinder surface $\Gamma_{\text {cyl }}$. We emphasize that the cylinder is assumed to be rigid, so there is no elastic deformation of the cylinder surface along its span. More generally, we assume that there is no dependence of the cylinder and NES parameters on the spanwise coordinate $z$.

We now comment on the nature of the coupling between the cylinder and the rotational NES, as it is essential to understanding the mechanisms by which the NES may be used as a passive energy harvesting and VIV suppression device. As noted above, the rectilinear motion of the cylinder and the rotational motion of the NES are inertially coupled, thus providing a mechanism for energy to be mechanically transferred to, and dissipated by, the NES (i.e., not only by the viscosity of the fluid). Inertial coupling appears nonlinearly in the rigid body equations (see the first terms on the righthand sides of $(2 \mathrm{a}, \mathrm{b}))$, and the strength of the nonlinearity is governed by the parameter $\varepsilon$. Rather than restricting our attention to small values of $\varepsilon(0<\varepsilon \ll 1)$ as is usually done in the context of weakly nonlinear coupling [11], we consider values of $\varepsilon$ that account for a significant fraction of the overall cylinder mass $(\varepsilon=\mathscr{O}(1))$. In addition to this, linearization of the coupling terms (i.e., the first term on the righthand side of (2a) and the right-hand side of (2b)) about any equilibrium position ( $y=0$ and $\theta=n \pi$, with $n$ an integer) yields no linear terms. This makes the inertial coupling between the NES and the cylinder essentially nonlinear [32]. Essential nonlinearity is a key attribute of the rotational NES since, lacking a preferential resonance frequency, it is capable of engaging in resonance capture with the cylinder (directly) and the surrounding fluid (indirectly) over broad ranges of excitation frequencies and energies. With the addition of weak dissipation, characterized by $\lambda$, the exchange of energy between the cylinder and the NES becomes nearly unidirectional, so the NES essentially absorbs kinetic energy from the cylinder motion in a nearly irreversible fashion. This process, termed targeted energy transfer (TET), confers the rotational NES the ability to act as an efficient vibration suppression device. This feature has been verified in a variety of engineering configurations [11]. 
(a)

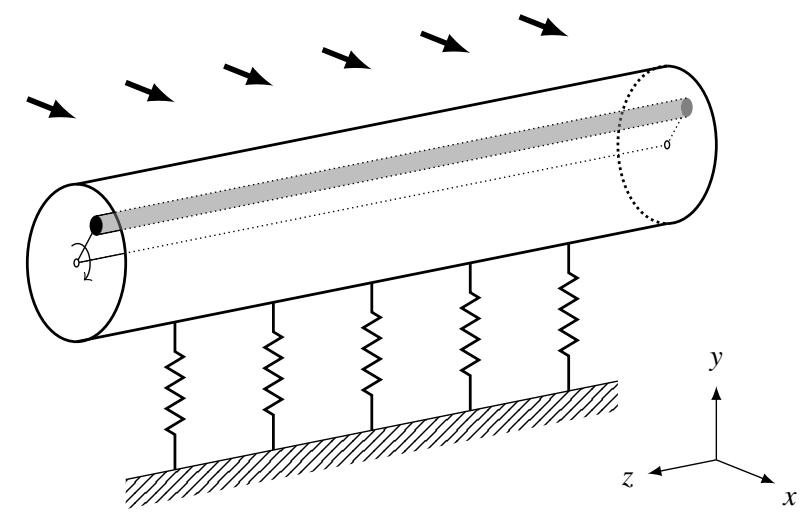

(b)

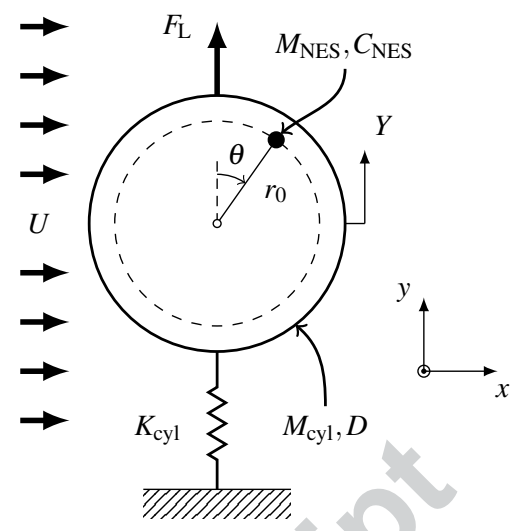

Fig. 1: (a) Perspective view, and (b) spanwise cross-section of a three-dimensional linearly-sprung circular cylinder in crossflow equipped with a rotational NES. In (a), the chain of springs should be thought of as a distributed stiffness along the span of the cylinder (with stiffness $K_{\text {cyl }}$ per unit length of the cylinder), and the eccentric NES "shaft" as a line mass (with mass $M_{\mathrm{NES}}$ per unit length) allowed to rotate about the cylinder axis. In (a) and (b), the "ground" to which the springs connect the cylinder should not be thought of as part of the boundary of the domain.

As discussed in $\S 1$, recent work by the authors and colleagues has established that a rotational NES can significantly alter the VIV of a sprung cylinder and the associated vortex shedding at low-to-intermediate Reynolds number $(20 \leq R e \leq 100)$. Some of the phenomena reported included passive VIV suppression, partial stabilization of the two-dimensional vortex street, and significant drag reduction, all of which were attributed to the sole presence of the rotational NES. In marine and estuarine currents, however, the flow is generally fully turbulent, with $R e$ well beyond 1500. At such high $R e$ values, virtually nothing is known about the effect that a rotational NES might have on the VIV and the flow. But there is hope that some of the results generated in the laminar regime may be used as a guide as we venture in the turbulent regime, for the following reason. Roshko [33] showed that the Strouhal number maintains a nearly constant value $(S t=0.19 \pm 0.02)$ over a wide range of $R e$ values that encompasses the laminar regime, and includes a significant part of the turbulent regime. As discussed by Roshko [33], significant aspects of vortex shedding by a cylinder (not limited to the Strouhal number) are qualitatively similar over that range. Thus, there is reason to believe that an understanding of the flow, of VIV, and of approaches to modifying VIV at $R e \leq 100$ can be adapted for use at much higher Re.

In what follows, we consider a value of $\operatorname{Re}(10000)$ comparable to those used in a series of experiments by Bernitsas and colleagues $[4,6]$, which we will use as reference. We set the dimensionless spring constant to $f_{\mathrm{n}}^{*}=0.2$, close to the shedding frequency $S t$ for flow past a fixed cylinder at $R e=10000$. The value of $f_{\mathrm{n}}^{*}$ is intentionally chosen close to St so as to evaluate the extent to which the NES is capable of altering large-amplitude VIV that occurs in the absence of NES. The cylinder and NES mass are initially motionless, the former at its equilibrium position, and the latter at $\theta(0)=\pi / 2$. Finally, following Blanchard et al. [34], we let $m^{*}=10$ and $\bar{r}_{0}=0.458$. We note that with this value of $\bar{r}_{0}$, the NES may lie inside the cylinder.

\subsection{Computational approach}

The computational solution is effected using the spectralelement code Nek5000 [35], whose use and adaptation to two-dimensional VIV configurations with and without NES have been described in $[27,26,34]$. In the present case, however, the flow and geometry are three-dimensional (3-D). The 3-D mesh is constructed by "extruding" a 2-D mesh along the spanwise direction. Specifically, we begin by generating a 2-D mesh with streamwise and cross-stream extents $L_{x}$ and $L_{y}$, respectively. We produce several copies of this 2-D mesh, which are then stacked along the spanwise direction to create a 3-D mesh with spanwise extent $L_{z}$. The number of 2-D layers along the span is given by $n_{z}+1$, where $n_{z}$ is the number of spectral elements in the spanwise direction. For simplicity, we use uniform spacing between each layer along the span. This process, whereby a 3-D mesh is generated by extrusion of a 2-D mesh on a given number of levels, takes full advantage of the tensor-product structure of 2-D meshes in Nek5000.

The "baseline" 2-D mesh that we use to construct the 3$\mathrm{D}$ mesh is composed of 704 elements, with dimensions $50 \mathrm{D}$ and $20 D$ in the streamwise and cross-stream directions, respectively (Fig. 2). The cylinder center is located $20 D$ from the inlet and equidistantly from the sidewalls. From this 2-D mesh, we construct by extrusion a 3-D mesh with 10 ele- 
ments in the $z$ direction $\left(n_{z}=10\right)$ spanning $L_{z}=8 D$ (Fig. 2). We use this 3-D mesh with 7040 elements in all of our production runs. We note that use of a sufficiently large domain is critical to minimize blockage effects, as they might lead to erroneous results [36]. For our 2-D and 3-D meshes, the resulting blockage, based on the cylinder diameter and the cross-stream extent of the domain, is found to be $2.5 \%$.
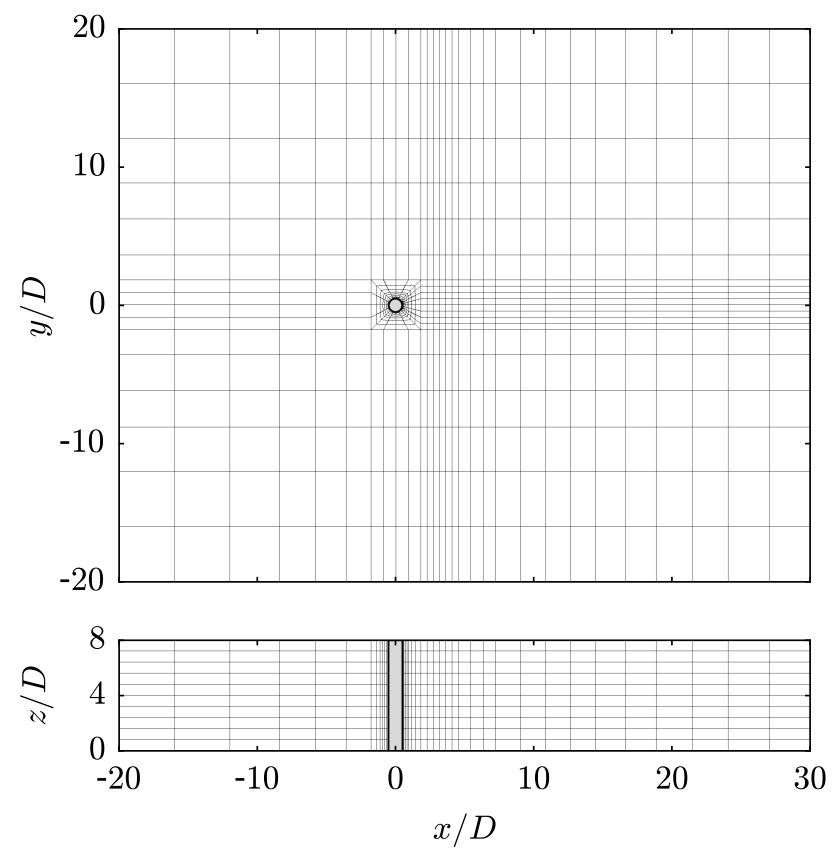

Fig. 2: Spectral-element discretization of an $(x, y)$-plane (top) and the $y=0$ plane (bottom). Each macro-element shown is further discretized using $N+1$ Gauss-LobattoLegendre quadrature points, where $N$ is the polynomial order.

At the "top" and "bottom" boundaries, we specify a symmetry (no-penetration) boundary condition equivalent to $\mathbf{v}$. $\mathbf{n}=0$ and $(\nabla \mathbf{v} \cdot \mathbf{t}) \cdot \mathbf{n}=0$, where $\mathbf{n}$ and $\mathbf{t}$ are the unit vectors normal and tangential to the side boundary, respectively. A stress-free boundary condition $(\boldsymbol{\sigma} \cdot \mathbf{n}=0)$ is enforced at the outlet. To facilitate development of three-dimensional effects in the wake, we allow the inlet velocity profile to be a user-specified function of $\tau, y$, and $z$, but require that it relax to uniform flow by some prescribed time $\tau_{\text {trans }}$ (i.e., $\mathbf{v}\left(x=x_{\text {inlet }}, y, z, \tau\right)=\mathbf{e}_{x}$ for all $\tau \geq \tau_{\text {trans }}$; in what follows, we take $\left.\tau_{\text {trans }}=25\right)$. We prescribe periodic boundary conditions for the velocity field in the spanwise direction. We do so in recognition of the fact that periodic boundary conditions appropriately model a cylinder of infinite length. We note that periodic boundary conditions have been widely used in 3-D computations of flow past a cylinder. How this choice affects the computational results is beyond the scope of the present work, but we mention that a number of studies have estab- lished its validity for 3-D flow past a fixed circular cylinder [37-39].

The no-slip boundary condition on the cylinder surface,

$\left.\mathbf{v}\right|_{\Gamma_{\mathrm{cyl}}}=\frac{\mathrm{d} y_{1}}{\mathrm{~d} \tau} \mathbf{e}_{y}$,

requires solving a moving-boundary problem. To this end, the Navier-Stokes equations are cast in an Arbitrary LagrangianEulerian (ALE) frame. Time integration of the fluid variables is performed using an unconditionally-stable time-stepping scheme based on backward-differentiation/extrapolation discretization formulas. The rigid-body motion is advanced using a fourth-order Runge-Kutta scheme. The rigid-body equations and the discretized Navier-Stokes equations, used to evaluate the lift coefficient, are solved in a staggered fashion; that is, we have assumed that, in the solution of the fluid-structure interaction problem, there is no need for equilibrating iterations in each time step, since the structure considered here is rigid, and provided that the time-step size is chosen small enough. This assumption was shown to be satisfactory in [42], who performed computations with the same fluid solver in which the fluid and structural responses at each time step were computed iteratively, with insignificant effects on the response of the system (compared to the solution obtained without equilibrating iterations).

In situations where the flow is expected to be fully turbulent, we use a filtering approach that subtracts a small amount of energy associated with the higher modes within each element [40]. The effect of the filter is to suppress high wavenumber instabilities, which are generally unphysical, and might lead the solution to blow up. Specifically, the $N$ th mode is damped by $5 \%$ and the $(N-1)$ th mode by $1.25 \%$. (Here, $N$ is the polynomial order, and the modal space is composed of the Legendre polynomials of degree $N$.) The number of modes to damp out and the damping factor associated with each damped mode should be determined on a case-by-case basis. This approach is in the same spirit as any large-eddy-simulation (LES) model, in which the smaller scales are filtered out through a similar procedure. We note that the filter does not inhibit spectral convergence, so its effect is nil when the solution is well resolved in space [40, 41].

In all that follows, the polynomial order is $N=5$, and the time-step size is $\Delta \tau=10^{-3}$. Validation of the computational approach is discussed in Appendix A.

\section{Results}

In this section, we investigate how a rotational NES can be used to enhance performance of hydrokinetic power generation by VIV. We consider several values of the mass ratio ( $\varepsilon=0.28$, and 0.33 ) and the dimensionless damping coefficient $(\lambda=0.09,0.1$, and 0.11$)$, so as to determine the extent 
to which small variations in $\varepsilon$ and $\lambda$ affect the statistics of the solution. We make no attempt to optimize the NES parameters toward maximal power efficiency, and leave this issue to future work.

The efficiency of the rotational NES is quantified by the (dimensional) peak power per unit length

$P_{\max }=\max _{T} C_{\mathrm{NES}}(\mathrm{d} \theta / \mathrm{d} t)^{2}$

and average power per unit length

$\bar{P}=\frac{1}{T} \int_{T} C_{\mathrm{NES}}(\mathrm{d} \theta / \mathrm{d} t)^{2} \mathrm{~d} t$,

computed over some time interval $T$. To facilitate comparison with the results by Bernitsas et al. [7], we rewrite $P_{\max }$ and $\bar{P}$ to make explicit the dependence on the combination parameter $\rho_{\mathrm{f}} D U^{3}$. Using the definitions of the dimensionless variables introduced in $\S 2.1$, we arrive at

$P_{\max }=\frac{\pi}{4} \varepsilon \bar{r}_{0}^{2} m^{*} P_{\max }^{*} \rho_{\mathrm{f}} D U^{3}$,

$\bar{P}=\frac{\pi}{4} \varepsilon \bar{r}_{0}^{2} m^{*} \bar{P}^{*} \rho_{\mathrm{f}} D U^{3}$

where we have defined the dimensionless peak power and average power per unit length as

$P_{\max }^{*}=\max _{T^{*}} \lambda \dot{\theta}^{2}$

$\bar{P}^{*}=\frac{1}{T^{*}} \int_{T^{*}} \lambda \dot{\theta}^{2} \mathrm{~d} \tau$,

respectively. Here, overdot denotes differentiation with respect to $\tau$, and $T^{*}=T U / D$. The above definitions rely on the assumption that all of the energy dissipated by the rotational damper of the NES can be harnessed with no loss. This assumption is consistent with prior work done in a similar context, in which an NES was used to harvest kinetic energy from an oscillating structure through electromagnetic coupling between the mechanical parts and a side electrical circuit $[43,20]$. We also note that $P_{\max }$ and $\bar{P}$ are proportional to the mass ratio $\varepsilon$ and the NES damping coefficient $\lambda$. For a higher value of $R e$ (close to $9.2 \times 10^{4}$ ), the VIVACE experiment by Bernitsas et al. [7] produced maximum peak power per unit length and averaged power per unit length of $P_{\text {max }, \text { VIVACE }}=0.154 \rho_{\mathrm{f}} D U^{3}$ and $\bar{P}_{\mathrm{VIVACE}}=0.11 \rho_{\mathrm{f}} D U^{3}$, respectively. There, the factors multiplying $\rho_{\mathrm{f}} D U^{3}$ were functions of the properties of the electrical circuit. By contrast, for VIV with rotational NES, the same factors in $(6 a, b)$ depend on the mechanical components of the NES.

In addition to power considerations, we also need to quantify transfers of energy between the fluid, the cylinder, and the NES. To this end, we define the energy dissipated by the NES damper as

$C_{\mathrm{diss}}=\int_{T_{C}} \lambda \dot{\theta}^{2} \mathrm{~d} \tau$ and the energy-transfer coefficient $C_{\mathrm{E}}$ as

$C_{\mathrm{E}}=\int_{T_{C}} C_{\mathrm{L}} \dot{y}_{1} \mathrm{~d} \tau$

where the interval of integration $T_{c}$ is chosen to be one cylinder cycle, defined as the elapsed time between two instants at which the cylinder center crosses the midplane $y=0$ with positive velocity. (When the response is not time-periodic, the value of $T_{c}$ varies from one cylinder cycle to the next.) The energy-transfer coefficient $C_{\mathrm{E}}$ quantifies the work done on the cylinder by the fluid over one cycle, and its sign provides information about the direction of energy transfer, with a positive sign indicating that the fluid does work on the cylinder and a negative sign indicating that the cylinder does work on the fluid. The sign of the mechanical energy transfer between the oscillating cylinder and the flow is critical, as it determines whether cross-flow oscillations tend to increase or decrease in amplitude. For example, the energytransfer coefficient has been successfully used to determine flow regimes that can be produced in NES-less VIV [44], and explain the significant wake elongation seen in NESequipped VIV at lower Reynolds number [34].

\subsection{Response for NES-less VIV}

We begin with a computation with $\varepsilon=0$ (corresponding to NES-less VIV), and $R e, m^{*}$ and $f_{\mathrm{n}}^{*}$ as discussed above. Figure 3 shows time series for $y_{1}, C_{\mathrm{L}}, C_{\mathrm{D}}$ and $C_{\mathrm{E}}$ in the statistically steady state. In Fig. $3 d$, and in all subsequent figures showing time series of $C_{\mathrm{E}}$, each dot corresponds to one cylinder cycle, and we choose to connect the dots with solid lines to facilitate visualization; those lines should not be thought of as the result of any kind of interpolation procedure of the data within a cylinder cycle. Figure 3 a shows that the maximum oscillation amplitude of $y_{1}$ at $R e=10000$ (about 0.835 ) is nearly twice as large as that for the limit cycle at $R e=100$ (about 0.497 ). The mean $\operatorname{drag} \bar{C}_{\mathrm{D}}$ at $R e=$ 10000 (about 2.209) is also much larger than at $R e=100$ (about 1.895). This observation is consistent with Nguyen $\&$ Tamarel [45], who noted that for a smaller value of the density ratio $\left(m^{*}=1.5\right)$, the value of $\bar{C}_{\mathrm{D}}$ in the lock-in region is close to 2.2 .

Figure 4 shows power spectra for $y_{1}, C_{\mathrm{L}}$, and the streamwise component of the velocity $v_{x, P}$ at a point located in the near wake. This point, referred to as Point $\mathrm{P}$, is located $3 D$ downstream of the cylinder center, $1 D$ above the midplane $y=0$, and equidistantly from the spanwise boundaries of the computational domain. The power spectrum for $v_{x, P}$ (Fig. 4c) is clearly broadband (with a slightly stronger contribution at $f D / U=0.2$ ), which is a manifestation of the turbulent nature of the flow. The power spectrum for $C_{\mathrm{L}}$ is also broadband, but the contribution at $f D / U=0.2$ is more pronounced than in Fig. 4c. The power spectrum 
(a)

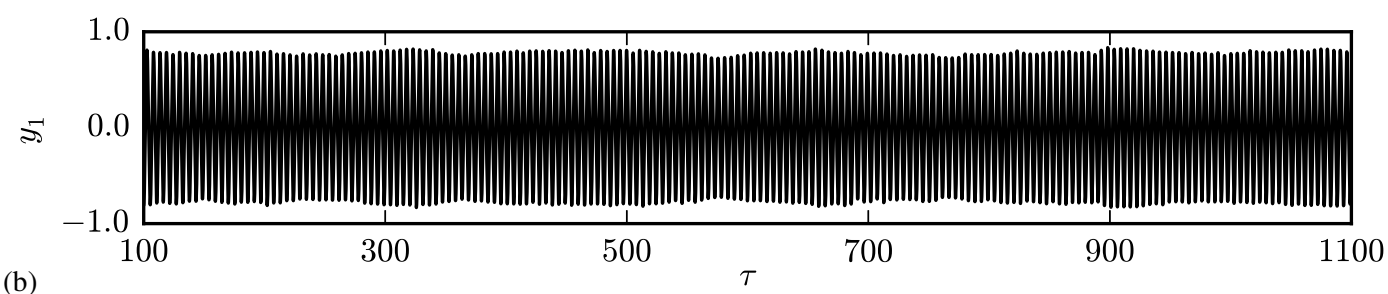

(b)

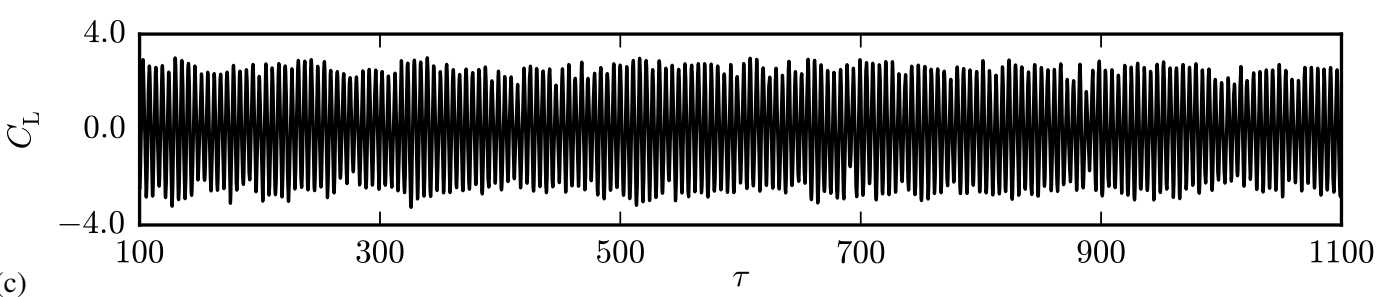

(c)

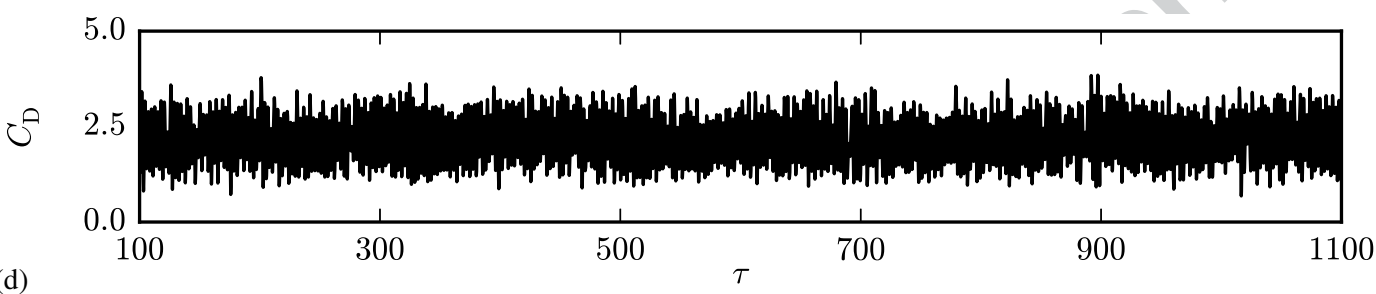

(d)

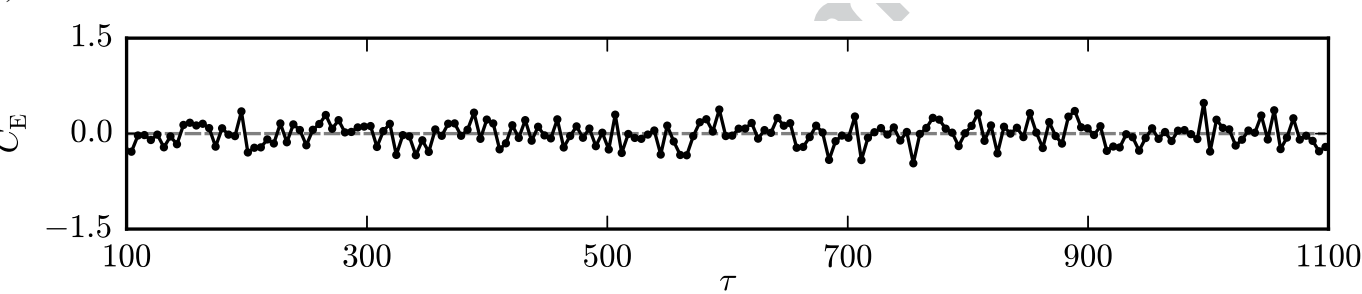

Fig. 3: For NES-less VIV at $R e=10000$, time series of (a) cylinder displacement, (b) lift coefficient, (c) drag coefficient, and (d) energy-transfer coefficient. The zero-level line in (d) is shown in dashed grey.

for $y_{1}$ shows a clear dominant frequency at $f D / U=0.2$, which corresponds to the natural frequency of the cylinder. Because the latter is close to the shedding frequency, some of the frequency contributions from the lift coefficient are "filtered out" by the cylinder equation (2a) and therefore appear much weaker in the spectrum of $y_{1}$.

Figure 5 shows the spatial distribution of the spanwise vorticity $\omega_{z}$ in the $z=4$ plane and the $y=0$ plane, along with an isosurface of the $\lambda_{2}$-criterion at $\tau=140.9$, at which time the cylinder crosses the midplane $y=0$ with negative velocity (Fig. 3a). The $\lambda_{2}$-criterion identifies vortices as flow regions in which

$\lambda_{2}\left(\mathbf{S}^{2}+\boldsymbol{\Omega}^{2}\right)<0$,

where $\mathbf{S}=\left(\nabla \mathbf{v}+\nabla \mathbf{v}^{\dagger}\right) / 2$ is the rate-of-strain tensor, $\boldsymbol{\Omega}=$ $\left(\nabla \mathbf{v}-\nabla \mathbf{v}^{\dagger}\right) / 2$ is the vorticity tensor, and $\lambda_{2}(\mathbf{A})$ denotes the intermediate eigenvalue of a symmetric tensor A [46]. Figure 5 illustrates the complexity of the flow at $R e=10000$. The vorticity distributions in Fig. 5a exhibit many smallscale structures in the near wake along the span of the cylin- der. Figure $5 b$ shows that these complex structures persist downstream as a result of advection.

Finally, we note that there is no distinct trend in the time series for $C_{\mathrm{E}}$ (Fig. 3d), in that the energy-transfer coefficient frequently changes sign as kinetic energy is exchanged between the cylinder and the flow. The fact that the vibration amplitude of the cylinder is large, and energy is constantly being transferred back and forth between the cylinder and the flow, suggests that a significant fraction of that energy could be harnessed by an appropriately designed NES. We explore this possibility below.

\subsection{Energy harvesting and vibration suppression in NES-equipped VIV}

We now consider the NES-equipped system. Figure 6 shows time series for NES parameters $\varepsilon=0.28$ and $\lambda=0.1$ (and the other parameters chosen as indicated above). These time series have several notable features. First, there is a considerable diminution of the oscillation amplitude of $y_{1}$, with a 
(a)

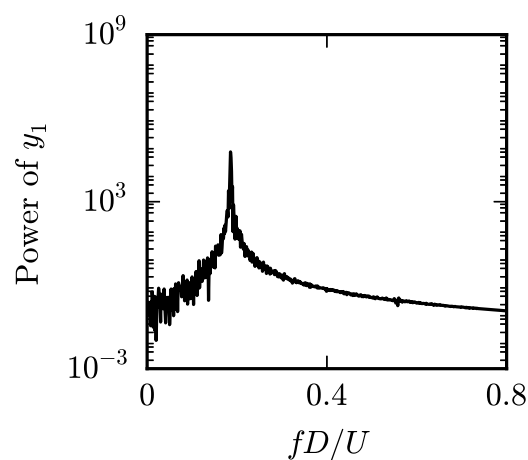

(b)

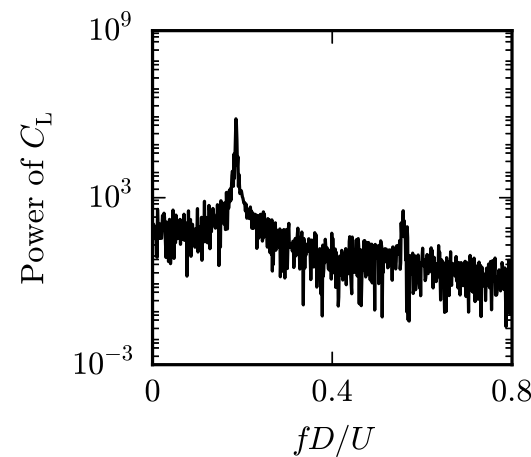

(c)

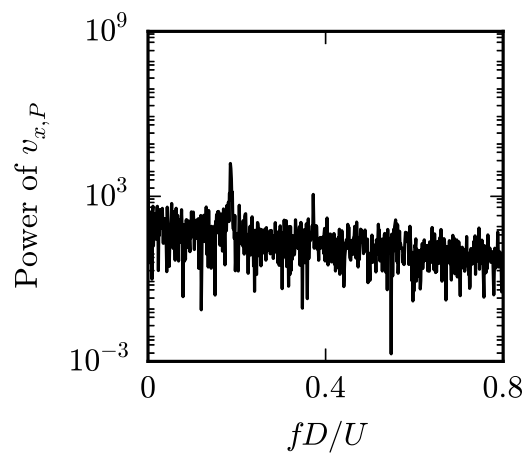

Fig. 4: Power spectra of (a) $y_{1}$, (b) $C_{\mathrm{L}}$, and (c) $v_{x, P}$ for NES-less VIV at $R e=10000$.

(a)

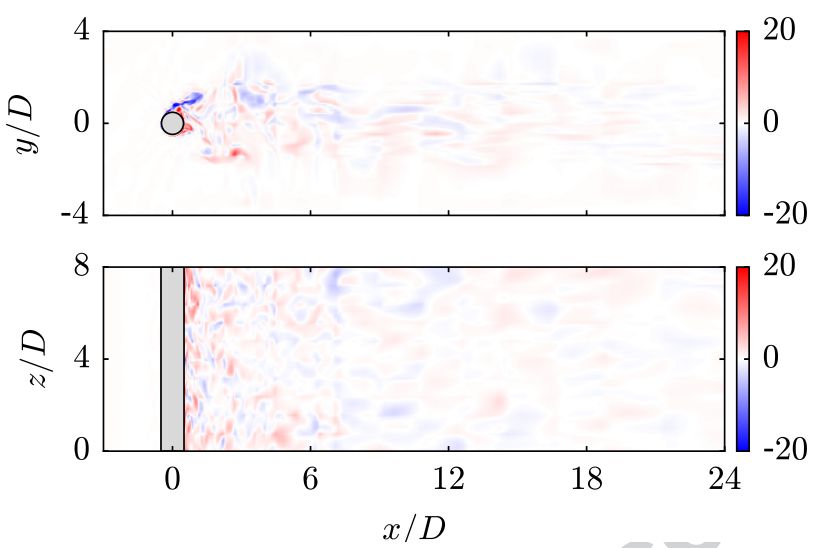

(b)

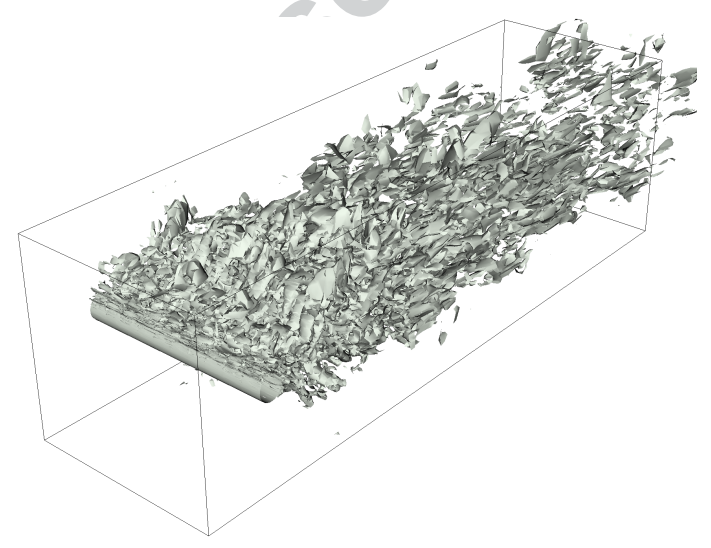

Fig. 5: For NES-less VIV at $R e=10000$, (a) slices of the spanwise vorticity distribution in the $z=4$ plane (top) and the $y=0$ plane (bottom), and (b) isosurface of the $\lambda_{2}$-criterion (with $\lambda_{2}=-0.1$ ). Snapshots are shown for $\tau=140.9$, at which time the cylinder crosses the midplane $y=0$ with negative velocity (cf. figure $3 \mathrm{a}$ ). The dimensions of the bounding box in (b) coincide with those of the visualization domain in (a), and are much smaller than those of the computational domain.

nearly two-fold reduction compared to Fig. 3a. The same is observed for the oscillation amplitude of $C_{\mathrm{L}}$. Second, Fig. 6e shows that the presence of the NES significantly affects the drag coefficient, whose average and maximum values appear to be only a fraction of what is seen when no NES is attached (cf. Fig. 3c).

Figure $6 \mathrm{a}$ also shows that the time series for $y_{1}$ features a number of "suppressed" intervals (for example, near $\tau=$ $250,380,516,772$, and 982), during which the cylinder executes very small-amplitude oscillations (with amplitude less than a tenth of the cylinder diameter). Each such suppressed episode is followed by growth in $y_{1}$, which is almost immediately suppressed afterwards. The physical mechanism is as follows. During each interval of growth of $y_{1}$, the angular velocity of the NES is quite low, and the energy-transfer coefficient is positive, indicating that growth in $y_{1}$ is caused by transfer of kinetic energy from the flow to the cylinder. As the oscillation amplitude of the cylinder grows, the NES is energized and enters a regime of nearly unidirectional rotation (cf. Figs. $6 \mathrm{~b}$ and $6 \mathrm{c}$ ), engaging in 1:1 resonance with the oscillating cylinder (i.e., the frequency of rotation of the NES is nearly equal to the frequency of rectilinear oscillation of the cylinder). This corresponds to TET from the rectilinear motion of the cylinder to the rotational motion of the NES. A significant fraction of kinetic energy is thereby dissipated by the linear viscous damper of the NES. The latter is able to passively suppress the flow-induced growth of the cylinder through targeted energy transfer. As the NES damper dissipates the surplus of energy, the cylinder motion quiets down, and the NES ultimately disengages from resonance.

This mechanism is evident from Fig. 6f, which shows that each large burst in $C_{\mathrm{E}}$ (corresponding to energy intake by the cylinder from the flow) is immediately followed by a peak in $C_{\mathrm{diss}}$ corresponding to dissipation of the energy transferred to the cylinder by the flow. Such behavior is typ- 
ical of quasi-unidirectional, irreversible TET in slowly modulated responses [11]. The number and succession of peaks in the time series for $C_{\mathrm{diss}}$, and the fact that those peaks are immediately preceded by a similar burst in $C_{\mathrm{E}}$, demonstrates that the rotational NES is capable of suppressing large-amplitudep VIV in turbulent flow. This is accompanied by considerable passive reduction in drag, which is particularly attractive for practical applications.

Examination of the wake structure in the suppressed intervals reveals another remarkable effect of the NES on the fluid-structure interaction dynamics. Figure 7 shows slices of the spanwise vorticity distribution and isosurface of the $\lambda_{2}$-criterion at $\tau=380$, at which time the amplitude of cylinder motion is much smaller than otherwise. Figure 7a shows that in the suppressed intervals, the vorticity in the near wake has smaller cross-stream extent than in the NES-less case (cf. Fig. 5a). More interestingly, the wake in the NES-equipped case appears more structured than in the NES-less case, with large coherent vortical structures clearly visible in the $y=0$ slice shown in Fig. 7a. These structures appear to span the entire length of the cylinder and alternate signs as they are advected downstream. For example, $\omega_{z}(x, y=0, z, \tau=480)$ is predominantly positive for all $z$ and for $x$ close to $8 D$, and predominantly negative for all $z$ and for $x$ close to $10 D$. This suggests that during the suppressed intervals, the effect of the NES is to partially re-laminarize the flow.

To determine whether the suppression mechanism shown in Fig. 6 is robust, we perform three additional computations for other values of $\varepsilon$ and $\lambda$. Rather than relying on cursory inspection of the time series, we compute the probability density function (PDF) for $y_{1}, C_{\mathrm{L}}$, and $C_{\mathrm{D}}$ over the time interval $100 \leq \tau \leq 1100$ (in the statistically steady state), which we compare to the NES-less case. Figure 8a shows that the tails in the PDF for $y_{1}$ are much wider in the absence of NES, which is proof that it is precisely the presence of the NES that causes significant VIV suppression. Figure $8 \mathrm{c}$ shows that, for the NES-equipped cases considered, most of the "mass" of the PDF for $C_{\mathrm{D}}$ shifts to lower values and is less spread out than for the NES-less case. This highlights the fact that the NES leads to substantial reduction in mean drag and drag amplitude.

We complement the PDFs shown in Fig. 8 with various statistics computed over the interval $100 \leq \tau \leq 1100$ for the NES-less and NES-equipped cases. Table 1 shows that the NES leads to significant VIV suppression and drag reduction in the four cases considered. We also note that the peak power per unit length extracted by the NES damper is comparable to that reported by Bernitsas et al. [7], suggesting that a large fraction of the kinetic energy associated with cylinder motion can be harnessed by the NES. As discussed above, this leads to reduction in the amplitude of $y_{1}$. However, the values for average power per unit length reported in Table 1 are about one and a half order of magnitude smaller than those of Bernitsas et al. [7]. We conjecture that this is because the recurrent suppressed episodes are interspersed by intervals during which the velocity of the NES is relatively small and, hence, not much kinetic energy is dissidepated by the rotational damper. This suggests that the average harvested power could in principle be much higher if the intervals of unidirectional rotation of the NES were to persist for a much larger number of convective time units, much like what had been seen for NES-equipped VIV at lower $R e$ [26]. This would require a sustained energy input from the flow to the cylinder, and would be the objective of a computational optimization study.

\section{Discussion}

The solutions reported for the NES-equipped system in $\$ 3.2$ are reminiscent of those found by Tumkur et al. [26] and Blanchard et al. [27] for the same system at much lower $R e$. For $R e=100, f_{\mathrm{n}}^{*}=0.167, m^{*}=10$, and a range of NES parameters, the authors in [26] and [27] reported the existence of solutions characterized by intervals of transient chaos alternating with quieter periods during which the cylinder motion slowly decays, the rotational NES executes nearly unidirectional rotational motion, and the drag decreases substantially. These intervals of regular motion have been referred to as "slowly decaying cycles". For $R e=100, f_{\mathrm{n}}^{*}=$ 0.167 and $m^{*}=10$, Blanchard et al. [27] conducted a parametric study of the slowly decaying solutions in the NES parameter space, and identified two mechanisms by which the system alternates between slowly decaying cycles and chaotic bursts. For small values of $\varepsilon$ (less than about 0.23 ), the slowly decaying cycles are shorter and dominated by a frequency close to $S t$, and the wake remains qualitatively similar to that of NES-less VIV. On the other hand, for values of $\varepsilon$ larger than about 0.23 , the slowly decaying cycles have much longer duration, and each ends with considerable elongation and symmetrization of the wake behind the cylinder. Wake elongation is accompanied by stabilization of the cylinder, which is nearly motionless at the end of each cycle (Figs. 9a,b).

The mechanism responsible for the slowly decaying solutions at $R e=100$ has been identified as successive resonance captures of the trajectory on an underlying slow invariant manifold (SIM) of the fluid-structure interaction dynamics [27]. During each slowly decaying cycle, the cylinder, the NES and the flow are in resonant motion (i.e., the time series for $y_{1}, \theta$, and $C_{\mathrm{L}}$ are dominated by a common frequency $\omega$ close to $S t$ ), which corresponds to capture of the trajectory on the SIM. The fact that resonance does not persist indefinitely and the trajectory eventually bursts into a regime of transient chaos is due to the topological structure of the SIM. The latter may be visualized in an amplitudephase plane, where the slowly varying amplitude of the cylin- 
der oscillation is denoted by $R$, and the slowly varying phase difference between the cylinder oscillation and the NES (nearly unidirectional) rotation by $\psi=\theta-\omega \tau$. Figure 9c shows that the SIM is composed of two branches symmetric about $\psi=-\pi$, with the right branch being stable, and the left branch being unstable. Superposition of the trajectory onto the SIM helps us understand the resonance mechanism. At the beginning of a slowly decaying cycle, the cylinder oscillation amplitude $R$ is quite large, and the trajectory is attracted to the stable branch of the SIM. As the slowly decaying motion sets in, the trajectory begins its descent along the stable branch of the SIM. This corresponds to gradual VIV suppression (Fig. 9a), wake elongation (Fig. 9b), and drag reduction. Theory predicts that the slowly decaying cycle should end when the trajectory reaches the lower end of the stable branch. At this point, the trajectory has nowhere to go because the other SIM branch is unstable, so it is expelled from the SIM and enters a regime of transient chaos. After some wandering about in phase space, a new resonance capture begins and the cycle repeats itself. We note that in practice, the trajectory leaves the SIM prematurely and never makes it to the lower folding point (Fig. 9c). This is because the SIM is derived from an asymptotic analysis in which only the leading-order terms are retained, so it is only a crude, low-dimensional approximation of the infinitedimensional dynamics. As a result, the SIM analysis fails to capture higher-order effects, and the early escape of the trajectory seen in the numerical experiments is an example of such.

Reviewing and understanding the intermittent suppression mechanism at $R e=100$ is useful because it facilitates interpretation of the results at $R e=10000$ discussed in \$3.2. Figure $6 \mathrm{~b}$ shows that there are many instances in the time series for $\theta$ in which the NES rotational motion has a pronounced preference in the direction of rotation for short times. For example, the NES rotates predominantly counter-clockwise immediately after $\tau=380$, and predominantly clockwise immediately after $\tau=516$. During these short intervals, the NES mass rotates more rapidly than otherwise (Fig. 6c), which in turn leads to salient peaks in $C_{\text {diss }}$ (Fig. 6f). This is almost always accompanied by a reduction in the amplitudes of $y_{1}$ and $C_{\mathrm{D}}$, very much like in the slowly decaying solutions found at $R e=100$. There are also clear changes in the wake at $R e=10000$, as the NES appears to cause partial relaminarization of the flow in the suppressed intervals (Fig. 7a). Of course, there is no clear slowly decaying cycle in the time series at $R e=10000$ shown in Fig. 6. There is also no alternation between periods of transient chaos and intervals of regular motion because the flow is turbulent and the rigid-body motion is chaotic. Yet, the striking similarities of the suppression mechanisms at $R e=100$ and $R e=10000$ lead us to conjecture that the VIV suppression seen at $R e=10000$ might be caused by successive short- lived resonance captures between the fluid flow, the cylinder and the NES. Verification of this conjecture requires an analysis similar to that performed by Blanchard et al. [27] at $R e=100$, in which changes should be made to account for the fact that the suppression episodes are much shorter at $R e=10000$ than at $R e=100$. As discussed in $\$ 3.2$, the original SIM analysis could be applied at $R e=10000$ if the intervals of nearly unidirectional NES rotation had much longer duration than those shown in Fig. 6b. Discovery of such dynamical regimes requires an extensive parametric search, so we leave this issue to future investigation.

\section{Conclusions}

The present work served as a preliminary study on the potential of a rotational NES for use as an energy harvesting device in submarine currents. We considered a value of $\operatorname{Re}(10000)$ at which flow past a linearly-sprung cylinder is three-dimensional and fully turbulent, and showed that a rotational NES is capable of harnessing a significant amount of kinetic energy from the motion of the cylinder, with performance comparable to previous experimental attempts. We also found that the rotational NES can lead to substantial suppression of cylinder oscillations, which was accompanied by a nearly two-fold reduction in drag. This result is significant, because it suggests that the NES may be able to considerably reduce drag in a range of other bluff-body flows, including flow past an airfoil or a tall building. The present study also provided evidence that the TET mechanism known to be realizable for two-dimensional flows at intermediate $R e$ carries over to three-dimensional turbulent flows at much higher $R e$.

We believe that this preliminary study, the first of its kind to focus on the effect of a passive internal rotational NES in three-dimensional turbulent VIV of a circular cylinder, provides sufficient motivation to pursue investigation of the fluid-structure interaction problem at high $R e$ values. Possible future research directions include gaining insight into the underlying dynamics governing the fluid-structure interaction, to reveal how the NES indirectly affects the surrounding flow. This will pave the way for predictive and optimized design of the NES for various applications. It is also critical to understand how the TET mechanism affects the rigid-body motion of the cylinder, the VIV and the wake. The ultimate goal is to show how TET can be employed for effective hydrodynamic energy harvesting and passive suppression of VIV in the turbulent regime.

Acknowledgements The authors gratefully acknowledge use of the facilities at the Argonne National Laboratory. The first author acknowledges the Computational Science and Engineering Fellowship program at the University of Illinois at Urbana-Champaign. This work was supported in part by National Science Foundation Grant CMMI-1363231. 
Any opinion, findings, and conclusions or recommendations expressed in this work are those of the authors, and do not necessarily reflect the views of the National Science Foundation.

\section{A Validation of the computational approach}

The computational approach is validated in two steps. We first compute flow past a fixed cylinder and a linearly-sprung cylinder with no NES at $R e=100$. At this Reynolds number, the flow is expected to be two-dimensional, laminar, and time-periodic. For the linearly-sprung case, we use parameters $f_{\mathrm{n}}^{*}=0.167$ and $m^{*}=10$ in order to facilitate comparison to previous results. Table 2 shows statistics computed with the 3-D mesh (with dimensions and number of elements given in \$2.2) for the fixed and linearly-sprung configurations. The statistics computed on the 3-D mesh are compared to values computed on the 2-D baseline mesh, as well as values reported in the literature for 2-D computations. The results in Table 2 validate the 3-D computational approach for nominally 2-D flows with and without mesh motion.

(a)

\begin{tabular}{lccc}
\hline & $S t$ & $C_{\mathrm{L}, \max }$ & $\bar{C}_{\mathrm{D}}$ \\
\hline Stansby \& Slaouti [47] & 0.166 & 0.350 & 1.320 \\
Anagnostopoulos [48] & 0.167 & 0.270 & 1.200 \\
Henderson [49] & 0.166 & 0.330 & 1.350 \\
Shiels et al. [50] & 0.167 & 0.300 & 1.300 \\
Tumkur et al. [26] & 0.167 & 0.340 & 1.381 \\
Present work: & & & \\
$\quad$ 2-D mesh & 0.170 & 0.342 & 1.392 \\
3-D mesh & 0.170 & 0.342 & 1.392 \\
\hline
\end{tabular}

(b)

\begin{tabular}{lcccc}
\hline & $S t$ & $C_{\mathrm{L}, \max }$ & $\bar{C}_{\mathrm{D}}$ & $y_{1, \max }$ \\
\hline Prasanth \& Mittal [36] & 0.164 & 0.190 & 1.880 & 0.503 \\
Fischer et al. [51] & 0.167 & 0.178 & 1.884 & 0.504 \\
Tumkur et al. [24] & 0.167 & - & - & 0.490 \\
Tumkur et al. [26] & 0.167 & - & - & 0.500 \\
Present work: & & & & \\
$\quad$ 2-D mesh & 0.167 & 0.161 & 1.895 & 0.497 \\
$\quad$ 3-D mesh & 0.167 & 0.161 & 1.895 & 0.497 \\
\hline
\end{tabular}

Table 2: Comparison of computed values at $R e=100$ for (a) flow past a fixed cylinder, and (b) flow past a linearlysprung cylinder.

The next step is to validate the computational approach in a situation where the flow is 3-D and fully turbulent. Because our production runs are at $R e=10000$, we choose that value for the convergence study as well. The amount of computational results available in the literature for transverse VIV at $R e=10000$ is vanishingly small, so we decide to benchmark our code against results for flow past a fixed cylinder at that Reynolds number. The statistics reported in table 3 show that the spectral-element framework guarantees adequate robustness of the results with respect to the computational parameters.

\begin{tabular}{lccc}
\hline & $S t$ & $C_{\mathrm{L}, \mathrm{rms}}$ & $\bar{C}_{\mathrm{D}}$ \\
\hline Bishop \& Hassan [52] & 0.201 & 0.463 & - \\
Gopalkrishnan [53] & 0.193 & 0.384 & 1.186 \\
Norberg [54] & 0.202 & 0.394 & - \\
Dong et al. [55] & 0.203 & 0.448 & 1.143 \\
$\begin{array}{l}\text { Present work: } \\
\quad \text { 3-D mesh }\end{array}$ & 0.193 & 0.398 & 1.229 \\
\hline
\end{tabular}

Table 3: Comparison of computed values at $R e=10000$ for flow past a fixed cylinder.

\section{Compliance with Ethical Standards}

Funding: This study was partially funded by National Science Foundation Grant CMMI-1363231. A. B. was partially supported by the Computational Science and Engineering Fellowship program at the University of Illinois at UrbanaChampaign.

Conflict of Interest: The authors declare that they have no conflict of interest.

\section{References}

1. Williamson, C.H.K., Govardhan, R.: Vortex-induced vibrations. Annual Review of Fluid Mechanics 36, 413-455 (2004)

2. Païdoussis, M.P., Price, S.J., De Langre, E.: Fluid-structure interactions: Cross-flow-induced instabilities. Cambridge University Press (2010)

3. Owen, J.C., Bearman, P.W., Szewczyk, A.A.: Passive control of VIV with drag reduction. Journal of Fluids and Structures 15, 597-605 (2001)

4. Bernitsas, M.M., Raghavan, K.: Reduction/suppression of VIV of circular cylinders through roughness distribution at $8 \times 10^{3}<$ $R e<1.5 \times 10^{5}$. In: ASME 2008 27th International Conference on Offshore Mechanics and Arctic Engineering, pp. 1001-1005 (2008)

5. Assi, G.R.S., Bearman, P.W., Kitney, N., Tognarelli, M.A.: Suppression of wake-induced vibration of tandem cylinders with freeto-rotate control plates. Journal of Fluids and Structures 26, 1045 1057 (2010)

6. Bernitsas, M.M., Raghavan, K., Ben-Simon, Y., Garcia, E.M.: VIVACE (Vortex Induced Vibration Aquatic Clean Energy): A new concept in generation of clean and renewable energy from fluid flow. Journal of Offshore Mechanics and Arctic Engineering 130, 041101 (2008)

7. Bernitsas, M.M., Ben-Simon, Y., Raghavan, K., Garcia, E.M.: The VIVACE converter: Model tests at high damping and Reynolds number around $10^{5}$. Journal of Offshore Mechanics and Arctic Engineering 131, 011102 (2009) 
8. Barrero-Gil, A., Pindado, S., Avila, S.: Extracting energy from vortex-induced vibrations: A parametric study. Applied Mathematical Modelling 36, 3153-3160 (2012)

9. Grouthier, C., Michelin, S., Bourguet, R., Modarres-Sadeghi, Y., De Langre, E.: On the efficiency of energy harvesting using vortex-induced vibrations of cables. Journal of Fluids and Structures 49, 427-440 (2014)

10. Ng, K.W., Lam, W.H., Ng, K.C.: 2002-2012: 10 years of research progress in horizontal-axis marine current turbines. Energies 6, $1497-1526(2013)$

11. Vakakis, A.F., Gendelman, O.V., Bergman, L.A., McFarland, D.M., Kerschen, G., Lee, Y.S.: Nonlinear targeted energy transfer in mechanical and structural systems. Springer Verlag, New York (2008)

12. Gendelman, O.V., Vakakis, A.F., Bergman, L.A., McFarland, D.M.: Asymptotic analysis of passive nonlinear suppression of aeroelastic instabilities of a rigid wing in subsonic flow. SIAM Journal on Applied Mathematics 70, 1655-1677 (2010)

13. Nucera, F., Iacono, F.L., McFarland, D.M., Bergman, L.A., Vakakis, A.F.: Application of broadband nonlinear targeted energy transfers for seismic mitigation of a shear frame: Experimental results. Journal of Sound and Vibration 313, 57-76 (2008)

14. Wierschem, N.E., Luo, J., Hubbard, S., Fahnestock, L.A., Spencer, B.F., Vakakis, A.F., Bergman, L.A.: Experimental testing of a large 9-story structure equipped with multiple nonlinear energy sinks subjected to an impulsive loading. In: Structures Congress 2013: Bridging Your Passion with Your Profession, pp. 22412252 (2013)

15. Bellet, R., Cochelin, B., Herzog, P., Mattei, P.O.: Experimental study of targeted energy transfer from an acoustic system to a nonlinear membrane absorber. Journal of Sound and Vibration 329, 2768-2791 (2010)

16. Quinn, D.D., Triplett, A.L., Vakakis, A.F., Bergman, L.A.: Energy harvesting from impulsive loads using intentional essential nonlinearities. Journal of Vibration and Acoustics 133, 011004 (2011)

17. Ahmadabadi, Z.N., Khadem, S.E.: Nonlinear vibration control and energy harvesting of a beam using a nonlinear energy sink and a piezoelectric device. Journal of Sound and Vibration 333, 44444457 (2014)

18. Zhang, Y., Tang, L., Liu, K.: Piezoelectric energy harvesting with a nonlinear energy sink. Journal of Intelligent Material Systems and Structures 28, 307-322 (2017)

19. Fang, Z.W., Zhang, Y.W., Li, X., Ding, H., Chen, L.Q.: Integration of a nonlinear energy sink and a giant magnetostrictive energy harvester. Journal of Sound and Vibration 391, 35-49 (2017)

20. Remick, K., Quinn, D.D., McFarland, D.M., Bergman, L., Vakakis, A.: High-frequency vibration energy harvesting from impulsive excitation utilizing intentional dynamic instability caused by strong nonlinearity. Journal of Sound and Vibration 370, 259279 (2016)

21. Mann, B.P., Sims, N.D.: Energy harvesting from the nonlinear oscillations of magnetic levitation. Journal of Sound and Vibration 319, 515-530 (2009)

22. Kremer, D., Liu, K.: A nonlinear energy sink with an energy harvester: Transient responses. Journal of Sound and Vibration 333, 4859-4880 (2014)

23. Quinn, D.D., Triplett, A.L., Bergman, L.A., Vakakis, A.F.: Comparing linear and essentially nonlinear vibration-based energy harvesting. Journal of Vibration and Acoustics 133, 011001 (2011)

24. Tumkur, R.K.R., Calderer, R., Masud, A., Pearlstein, A.J., Bergman, L.A., Vakakis, A.F.: Computational study of vortexinduced vibration of a sprung rigid circular cylinder with a strongly nonlinear internal attachment. Journal of Fluids and Structures 40, 214-232 (2013)

25. Tumkur, R.K.R., Domany, E., Gendelman, O.V., Masud, A., Bergman, L.A., Vakakis, A.F.: Reduced-order model for laminar vortex-induced vibration of a rigid circular cylinder with an internal nonlinear absorber. Communications in Nonlinear Science and Numerical Simulation 18, 1916-1930 (2013)

26. Tumkur, R.K.R., Pearlstein, A.J., Masud, A., Gendelman, O.V., Blanchard, A.B., Bergman, L.A., Vakakis, A.F.: Effect of an internal nonlinear rotational dissipative element on vortex shedding and vortex-induced vibration of a sprung circular cylinder. Journal of Fluid Mechanics 828, 196-235 (2017)

27. Blanchard, A.B., Gendelman, O.V., Bergman, L.A., Vakakis, A.F.: Capture into slow-invariant-manifold in the fluid-structure dynamics of a sprung cylinder with a nonlinear rotator. Journal of Fluids and Structures 63, 155-173 (2016)

28. Blanchard, A.B., Bergman, L.A., Vakakis, A.F., Pearlstein, A.J. Coexistence of multiple long-time solutions for two-dimensional laminar flow past a linearly-sprung circular cylinder with a rotational nonlinear energy sink (2018). Submitted to Physical Review Fluids

29. Mehmood, A., Nayfeh, A.H., Hajj, M.R.: Effects of a non-linear energy sink (NES) on vortex-induced vibrations of a circular cylinder. Nonlinear Dynamics 77, 667-680 (2014)

30. Dai, H.L., Abdelkefi, A., Wang, L.: Vortex-induced vibrations mitigation through a nonlinear energy sink. Communications in Nonlinear Science and Numerical Simulation 42, 22-36 (2017)

31. Dongyang, C., Abbas, L.K., Guoping, W., Xiaoting, E., Marzocca, P.: Numerical study of flow-induced vibrations of cylinders under the action of nonlinear energy sinks (NESs). Nonlinear Dynamics pp. 1-33 (2018)

32. Sigalov, G., Gendelman, O.V., Al-Shudeifat, M.A., Manevitch, L.I., Vakakis, A.F., Bergman, L.A.: Resonance captures and targeted energy transfers in an inertially-coupled rotational nonlinear energy sink. Nonlinear Dynamics 69, 1693-1704 (2012)

33. Roshko, A.: On the development of turbulent wakes from vortex streets. NACA TN 2913 (1954)

34. Blanchard, A., Bergman, L.A., Vakakis, A.F.: Targeted energy transfer in laminar vortex-induced vibration of a sprung cylinder with a nonlinear dissipative rotator. Physica D: Nonlinear Phenomena 350, 26-44 (2017)

35. Fischer, P.F., Lottes, J.W., Kerkemeier, S.G.: nek5000 Web page (2008). http://nek5000.mcs .anl.gov

36. Prasanth, T.K., Mittal, S.: Effect of blockage on free vibration of a circular cylinder at low $R e$. International Journal for Numerical Methods in Fluids 58, 1063-1080 (2008)

37. Braza, M., Faghani, D., Persillon, H.: Successive stages and the role of natural vortex dislocations in three-dimensional wake transition. Journal of Fluid Mechanics 439, 1-41 (2001)

38. Dong, S., Karniadakis, G.E.: DNS of flow past a stationary and oscillating cylinder at $R e=10000$. Journal of Fluids and Structures 20, 519-531 (2005)

39. Pontaza, J.P., Chen, H.C.: Three-dimensional numerical simulations of circular cylinders undergoing two degree-of-freedom vortex-induced vibrations. Journal of Offshore Mechanics and Arctic Engineering 129, 158-164 (2007)

40. Fischer, P., Mullen, J.: Filter-based stabilization of spectral element methods. Comptes Rendus de l'Académie des Sciences Series I: Mathematics 332, 265-270 (2001)

41. Boyd, J.P.: Two comments on filtering (artificial viscosity) for Chebyshev and Legendre spectral and spectral element methods. Journal of Computational Physics 143, 283-288 (1998)

42. Tumkur, R.K.R.: Modal interactions and targeted energy transfers in laminar vortex-induced vibrations of a rigid cylinder with strongly nonlinear internal attachments. Ph.D. thesis, University of Illinois at Urbana-Champaign (2014)

43. Barton, D.A.W., Burrow, S.G., Clare, L.R.: Energy harvesting from vibrations with a nonlinear oscillator. Journal of Vibration and Acoustics 132, 021009 (2010)

44. Leontini, J.S., Stewart, B.E., Thompson, M.C., Hourigan, K.: Wake state and energy transitions of an oscillating cylinder at low Reynolds number. Physics of Fluids 18, 067101 (2006) 
45. Nguyen, L.T.T., Temarel, P.: Numerical simulation of an oscillating cylinder in cross-flow at a Reynolds number of 10,000: Forced and free oscillations. In: ASME 2014 33rd International Conference on Ocean, Offshore and Arctic Engineering, p. V002T08A022. American Society of Mechanical Engineers (2014)

46. Jeong, J., Hussain, F.: On the identification of a vortex. Journal of fluid mechanics 285, 69-94 (1995)

47. Stansby, P.K., Slaouti, A.: Simulation of vortex shedding including blockage by the random-vortex and other methods. International Journal for Numerical Methods in Fluids 17, 1003-1013 (1993)

48. Anagnostopoulos, P.: Numerical investigation of response and wake characteristics of a vortex-excited cylinder in a uniform stream. Journal of Fluids and Structures 8, 367-390 (1994)

49. Henderson, R.D.: Details of the drag curve near the onset of vortex shedding. Physics of Fluids 7, 2102-2104 (1995)

50. Shiels, D., Leonard, A., Roshko, A.: Flow-induced vibration of a circular cylinder at limiting structural parameters. Journal of Fluids and Structures 15, 3-21 (2001)

51. Fischer, P., Schmitt, M., Tomboulides, A.: Recent developments in spectral element simulations of moving-domain problems. In: Recent Progress and Modern Challenges in Applied Mathematics, Modeling and Computational Science, pp. 213-244. Springer, New York (2017)

52. Bishop, R.E.D., Hassan, A.Y.: The lift and drag forces on a circular cylinder in a flowing fluid. Proceedings of the Royal Society of London A: Mathematical, Physical and Engineering Sciences 277, 32-50 (1964)

53. Gopalkrishnan, R.: Vortex-induced forces on oscillating bluff cylinders. Tech. rep., Woods Hole Oceanographic Institution, MA (1993)

54. Norberg, C.: Fluctuating lift on a circular cylinder: Review and new measurements. Journal of Fluids and Structures 17, 57-96 (2003)

55. Dong, S., Karniadakis, G.E., Ekmekci, A., Rockwell, D.: A combined direct numerical simulation-particle image velocimetry study of the turbulent near wake. Journal of Fluid Mechanics 569, 185-207 (2006) 
(a)
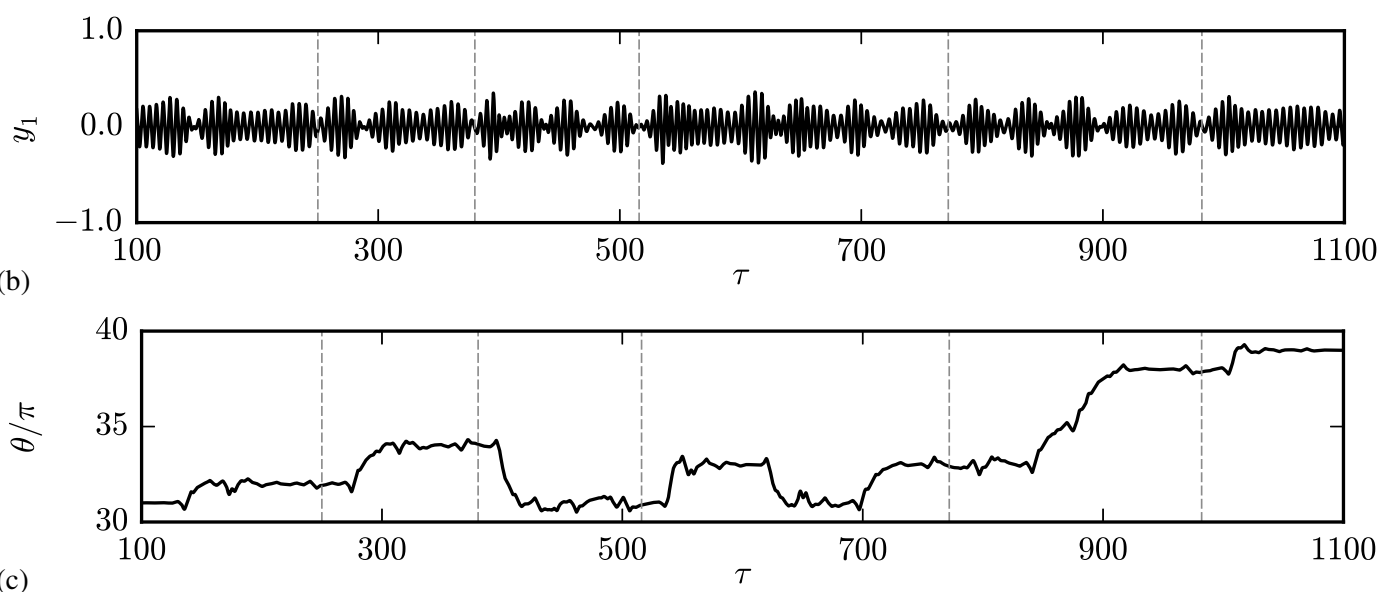

(c)

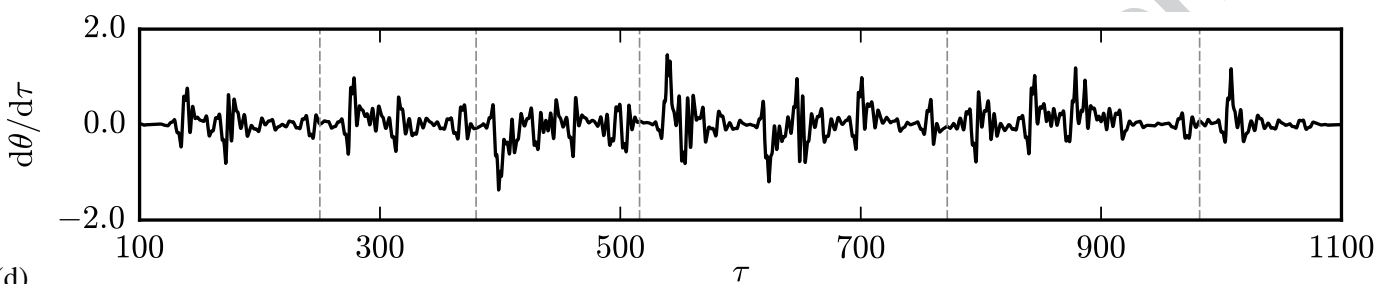

(d)

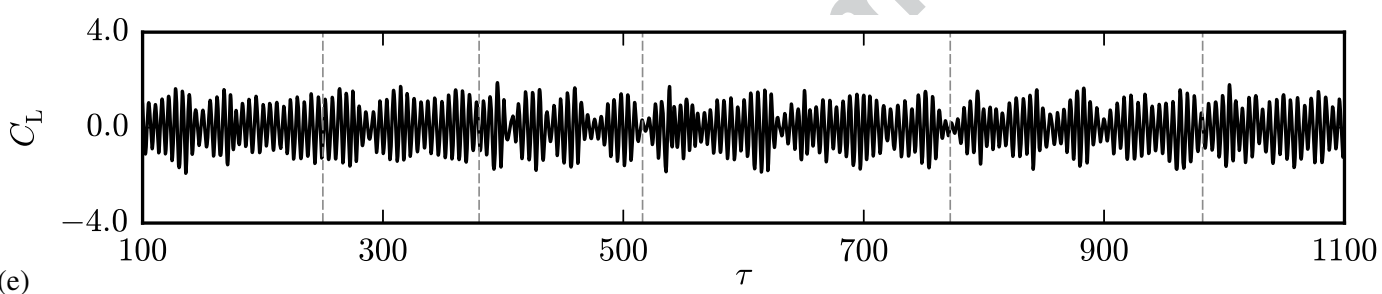

(e)

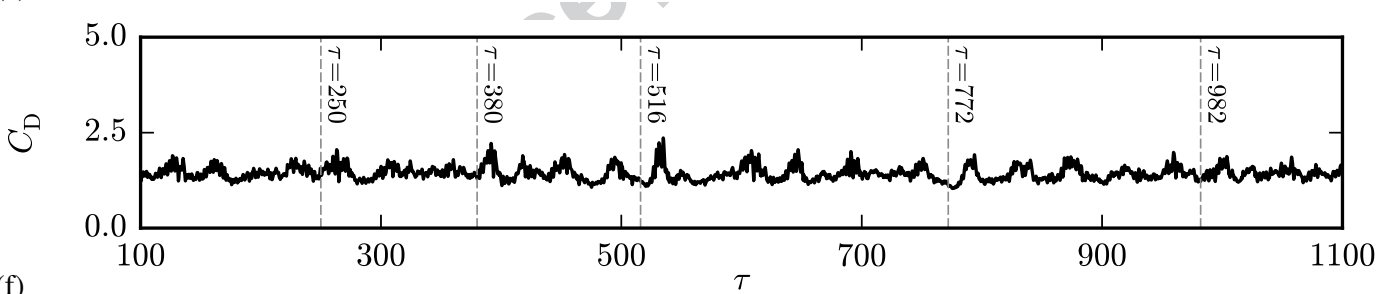

(f)

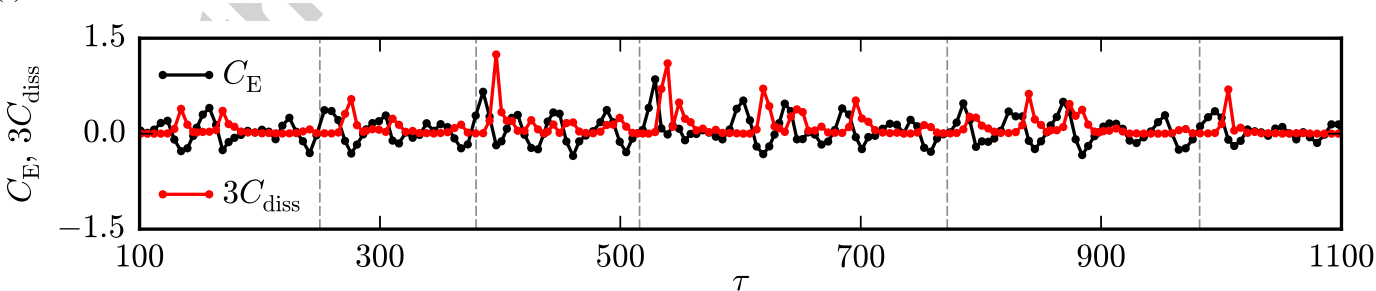

Fig. 6: For NES-equipped VIV at $R e=10000$ with NES parameters $\varepsilon=0.28$ and $\lambda=0.1$, time series of (a) cylinder displacement, (b) NES angular displacement, (c) NES angular velocity, (d) lift coefficient, (e) drag coefficient, and (f) energytransfer coefficient (black) and energy dissipated by NES damper (red). 
(a)

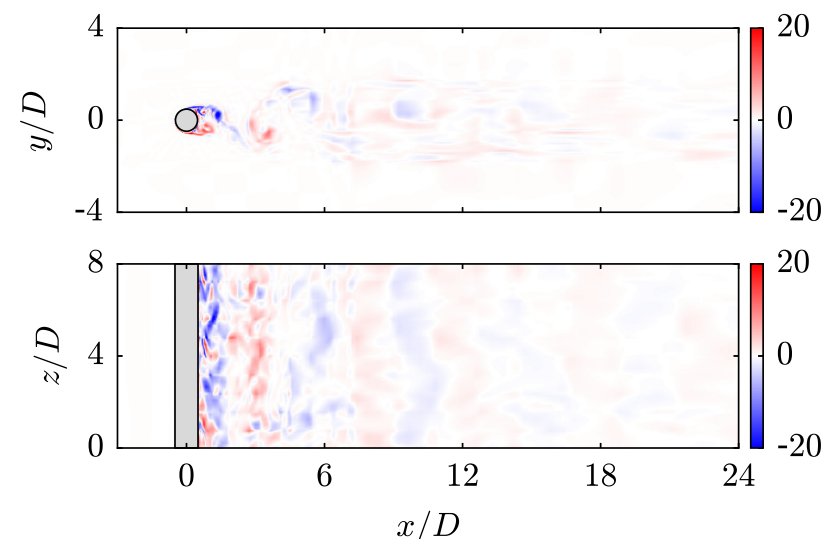

(b)

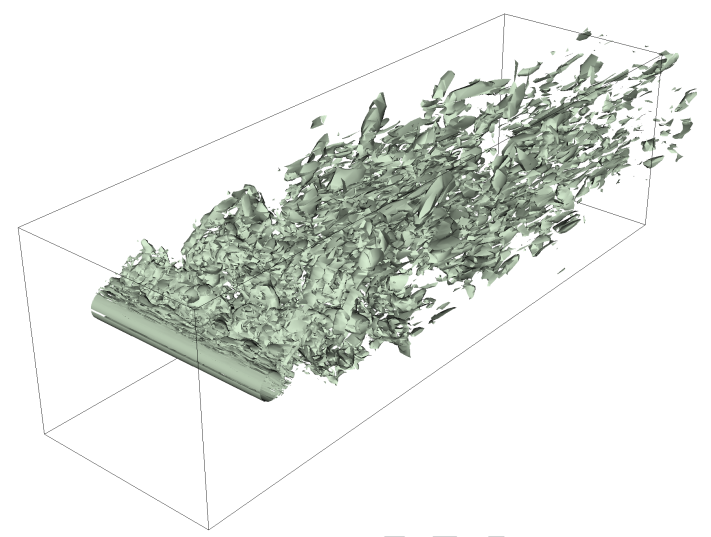

Fig. 7: For NES-equipped VIV at $R e=10000$ with NES parameters $\varepsilon=0.28$ and $\lambda=0.1$, (a) slices of the spanwise vorticity distribution in the $z=4$ plane (top) and the $y=0$ plane (bottom), and (b) isosurface of the $\lambda_{2}$-criterion (with $\lambda_{2}=-0.1$ ). Snapshots are shown for $\tau=380$ (cf. Fig. 6). The dimensions of the bounding box in (b) coincide with those of the visualization domain in (a), and are much smaller than those of the computational domain.

(a)

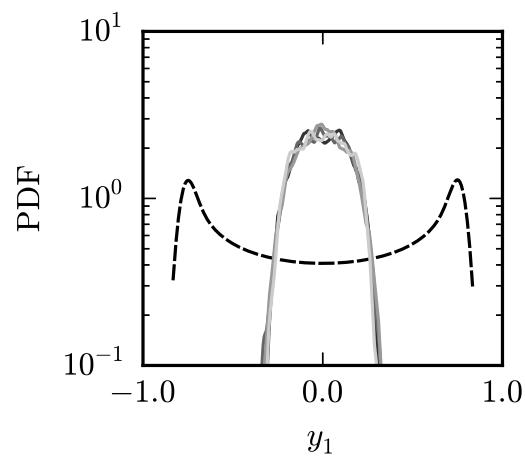

(b)

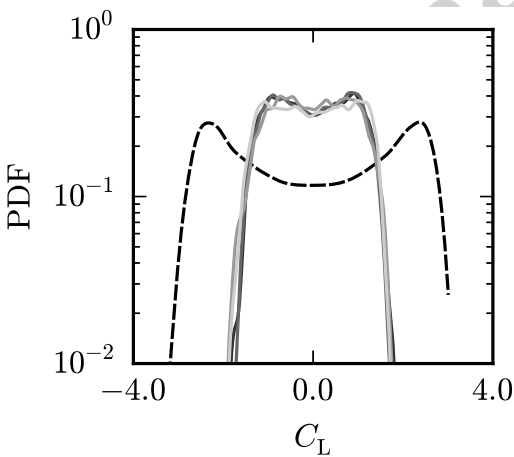

(c)

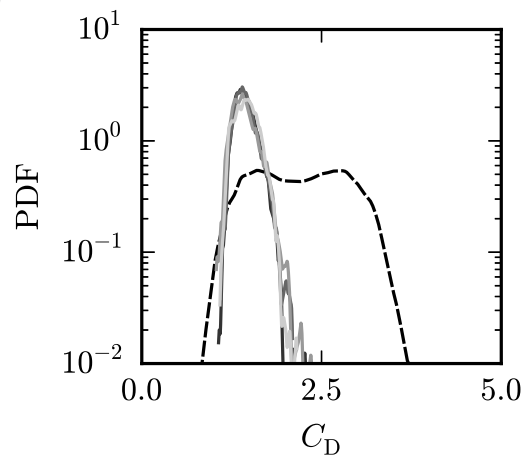

Fig. 8: Probability density function of (a) $y_{1}$, (b) $C_{\mathrm{L}}$, and (c) $C_{\mathrm{D}}$ at $R e=10000$. Dashed black: NES-less VIV; solid grey: NES-equipped VIV (from darker to lighter: $\varepsilon=0.28$ and $\lambda=0.1, \varepsilon=0.33$ and $\lambda=0.09, \varepsilon=0.33$ and $\lambda=0.1$, and $\varepsilon=0.33$ and $\lambda=0.11$.)

\begin{tabular}{cc|cccccc}
\hline \multicolumn{2}{c|}{ NES parameters } & \multicolumn{5}{c}{ Statistics } \\
$\varepsilon$ & $\lambda$ & $y_{1, \max }$ & $C_{\mathrm{L}, \max }$ & $C_{\mathrm{L}, \mathrm{rms}}$ & $\bar{C}_{\mathrm{D}}$ & $P_{\max } /\left(\rho_{\mathrm{f}} D U^{3}\right)$ & $\bar{P} /\left(\rho_{\mathrm{f}} D U^{3}\right)$ \\
\hline 0 & 0 & 0.8349 & 3.2609 & 1.8387 & 2.2090 & - & - \\
0.28 & 0.10 & 0.3702 & 1.8518 & 0.8381 & 1.4445 & 0.0881 & 0.0023 \\
0.33 & 0.09 & 0.3635 & 1.8211 & 0.8312 & 1.4480 & 0.1084 & 0.0081 \\
0.33 & 0.10 & 0.3826 & 1.9172 & 0.8282 & 1.4375 & 0.1045 & 0.0047 \\
0.33 & 0.11 & 0.3352 & 1.8823 & 0.8729 & 1.4608 & 0.1017 & 0.0029 \\
\hline
\end{tabular}

Table 1: Statistics for flow past linearly-sprung cylinder at $R e=10000$ with and without rotational NES. 
(a)

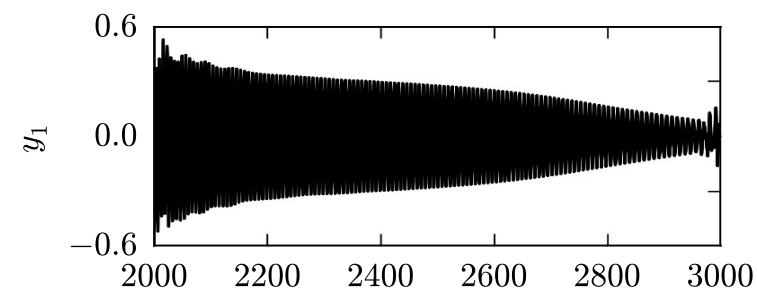

(b)

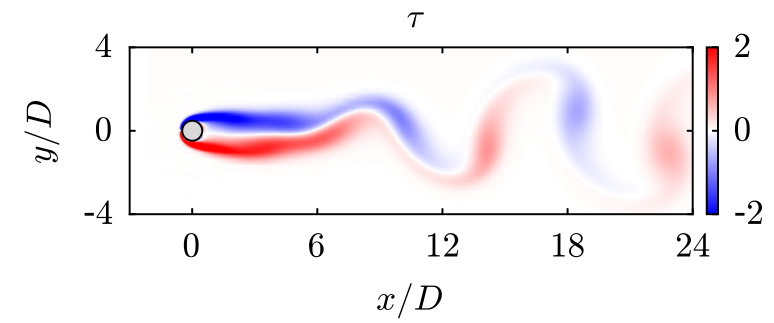

(c)

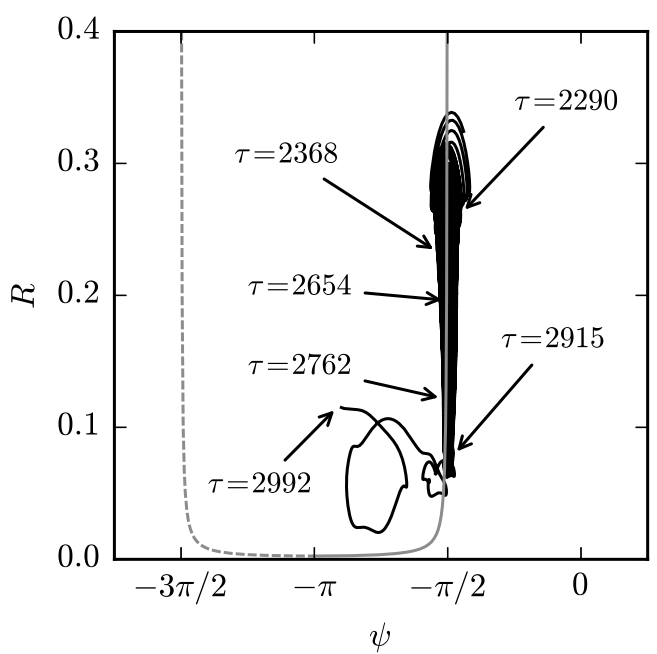

Fig. 9: For 2-D flow past an NES-equipped linearly-sprung cylinder at $R e=100$ with parameters $f_{\mathrm{n}}^{*}=0.167$ and $m^{*}=10$, and NES parameters $\varepsilon=0.33, \lambda=0.002745$ and $\bar{r}_{0}=0.458$, (a) time series for $y_{1}$ during a slowly decaying cycle, (b) vorticity distribution at $\tau=2947.7$, and (c) illustration of the transient resonance capture on the SIM for the time series shown in (a). In (c), the trajectory is shown in black, and the stable and unstable branches of the SIM are shown with solid and dashed grey lines, respectively. 